\title{
LA PRÁCTICA PEDAGÓGICA METACOGNITIVA COMO CATEGORÍA EMERGENTE EN UN MUNDO PERMEADO POR LA COMPLEJIDAD
}

Pájaro Manjarrés, Milton

LA PRÁCTICA PEDAGÓGICA METACOGNITIVA COMO CATEGORÍA EMERGENTE EN UN MUNDO PERMEADO POR LA COMPLEJIDAD

PANORAMA, vol. 14, núm. 26, 2020

Politécnico Grancolombiano, Colombia

Disponible en: http://www.redalyc.org/articulo.oa?id=343963784008

DOI: https://doi.org/10.15765/pnrm.v14i26.1484

Esta obra está bajo una Licencia Creative Commons Atribución-NoComercial-SinDerivar 4.0 Internacional. 
Artículos de investigación científica y tecnológica

\title{
LA PRÁCTICA PEDAGÓGICA METACOGNITIVA COMO CATEGORÍA EMERGENTE EN UN MUNDO PERMEADO POR LA COMPLEJIDAD
}

\author{
METACOGNITIVE PEDAGOGICAL PRÁCTICE \\ AS AN EMERGENT CATEGORY IN A WORLD \\ PERMEATED BY COMPLEXITY \\ PRÁTICA PEDAGÓGICA METACOGNITIVA COMO \\ UMA CATEGORIA EMERGENTE EM UM MUNDO \\ PERMEADO PELA COMPLEXIDADE \\ Milton Pájaro Manjarrés miltonpama@gmail.com \\ Universidad Simón Bolívar., Colombia
}

PANORAMA, vol. 14, núm. 26, 2020

Politécnico Grancolombiano, Colombia

Recepción: 26 Julio 2018

Aprobación: 14 Febrero 2020

DOI: https://doi.org/10.15765/ pnrm.v14i26.1484

Redalyc: http://www.redalyc.org/ articulo.oa?id=343963784008

\section{BY-NC-ND}

Resumen: La práctica pedagógica, entendida como una práctica social que adquiere sentido por medio de la interacción entre los sujetos y entre estos y su entorno, debe partir del carácter complejo que caracteriza al mundo en el que vivimos. En este sentido, resultaría insuficiente y hasta ilógico pensar en una práctica pedagógica estática, lineal y simplista. Por el contrario, la realidad de nuestro entorno insta de prácticas pedagógicas de carácter emergente, e incluso irreverente, que tengan como ejes centrales el dinamismo, la investigación, la reflexión, la dialogicidad, que religados con los principios de la metacognición formen individuos capaces de auto-dirigir sus propios procesos de aprendizaje y capaces de vivir y desenvolverse en una sociedad permeada por la complejidad.

Palabras clave: Práctica pedagógica, metacognición, complejidad, dialogicidad, reflexión.

Abstract: The pedagogical practice understood as a social practice that acquires meaning through the interaction among people and among them and their environment, must consider the complex nature that characterizes the world where we live. In this sense, it would be insufficient and even illogical to think of a static, linear and simplistic pedagogical practice. On the contrary, the reality of our environment calls for pedagogical practices with an emergent and even irreverent nature, that have dynamism, research, reflection and dialogue as central axes, which joined to the principles of metacognition educate human beings who are able to self-direct their own learning process and live well in a society permeated by complexity.

Keywords: Pedagogical practice, metacognition, complexity, dialogicity, reflection.

Resumo: A prática pedagógica entendida como uma prática social que adquire sentido através da interação entre os sujeitos e entre eles e seu ambiente, deve partir do caráter complexo que caracteriza o mundo em que vivemos. Neste sentido, seria insuficiente e até ilógico pensar em uma prática pedagógica estática, linear e simplista. Pelo contrário, a realidade de nosso ambiente exige práticas pedagógicas de natureza emergente e até irreverente que têm como eixos centrais o dinamismo, a pesquisa, a reflexão e o diálogo, que, ligados aos princípios da metacognição, formam indivíduos capazes de autodirigir seus próprios processos de aprendizagem e capazes de viver e desenvolver-se em uma sociedade permeada pela complexidade. 
Palavras-chave: Prática pedagógica, metacognição, complexidade, dialogalidade, reflexão.

\author{
"La educación verdadera es praxis, reflexión \\ $y$ acción del hombre sobre el mundo para \\ transformarlo" \\ Fuente: Paulo Freire
}

El concepto practica pedagógica tiene un carácter complejo y, en algunos casos, hasta controversial. Abordar este concepto requiere considerar elementos como los modelos pedagógicos, la diversidad de conceptos provenientes de la pedagogía, además de las particularidades de la sociedad y las instituciones donde se desarrollan dichas prácticas. Esta realidad le da un carácter complejo a la práctica pedagógica, lo que la convierte en un concepto nada fácil de definir. En este artículo se intentará definir el concepto de práctica pedagógica, llegando incluso a mirarla desde una mirada emergente que se presenta como práctica pedagógica metacognitiva.

Antes de llegar a esta conceptualización, se intentará establecer una diferenciación entre las concepciones que existen de la práctica docente y la práctica pedagógica, identificando puntos de divergencia entre éstas.

\title{
Práctica docente vs. práctica pedagógica
}

Resulta muy común utilizar los términos práctica docente y práctica pedagógica para referirse a lo mismo. Sin embargo, ambos conceptos corresponden a categorías distintas que, si bien tienen algunos puntos de convergencia, deben ser explicados, comprendidos y utilizados de manera diferente, dado los contrastes que se pueden establecer entre ambos conceptos.

Iniciando con el concepto de práctica docente, este se refiere principalmente a la labor que realiza el maestro en una institución educativa, a la acción de orientar un proceso de formación, de enseñar y a todas las condiciones que jurídica y administrativamente hacen parte de esta labor (Rockwell \& Mercado, 1989). Ahora bien, tal como se mencionó en el apartado anterior, la realidad del mundo tiene un carácter complejo y trasciende los límites en los que se enmarca lo que hasta aquí se entiende como práctica docente.

Según Cerdá (2001), la práctica docente se fundamenta desde dos enfoques principales: la práctica y la docencia. En primera instancia, enfatizar en la práctica hace que la labor del maestro no sea reducida solo a la enseñanza. Por otro lado, la docencia tiene que ver con ese carácter cotidiano de la práctica y todos los elementos que hacen parte de la misma y que intervienen en este proceso, tales como la interacción entre los sujetos, las necesidades, el contexto, entre otros.

El aporte más significativo de Cerdá (2001), se centra en considerar la práctica docente como una actividad que tiene en su eje principal el 
trabajo del maestro como tal, su accionar, el cual desarrolla en la escuela $\mathrm{u}$ otro espacio de formación como parte de unos intereses particulares a nivel social, político y, en algunos casos, hasta culturales (Rockwell \& Ezpeleta, 1986).

\section{¿Qué hace a la práctica pedagógica diferente de la práctica docente?}

La conceptualización que se hace en muchas ocasiones del término práctica docente y práctica pedagógica tiende a confundirse y es esa quizás la razón principal para que estos dos conceptos sean usados indistintamente y de manera errada por muchas personas. La labor del docente en la escuela o acción pedagógica, tal como lo dice Bernstein (1998), involucra un análisis de las interacciones que se pueden establecer desde lo que se denomina comunicación pedagógica (relación que se establece entre un individuo y los diferentes elementos que intervienen en el proceso educativo). Estas relaciones pedagógicas corresponden a lo que en sí se denomina práctica pedagógica.

Se puede decir, entonces, que la práctica pedagógica es una práctica con una esencia netamente social y, como toda práctica de esta naturaleza, tiene un carácter complejo que involucra elementos que van desde un nivel macro hasta llegar a un nivel micro (Giddens, 1986; Bourdieu, 1987).

A nivel macro, la práctica pedagógica la integran una serie de superestructuras; a nivel micro, se refiere a los ejes principales de la práctica que son los sujetos $\mathrm{y}$, a nivel meso, involucra todas aquellos elementos que fungen como mediadores entre lo macro y lo micro.

\section{¿Qué se entiende por práctica pedagógica?}

Hablar de práctica pedagógica es hablar de una categoría que fue formulada con el fin de recuperar la historia de la pedagogía durante los años 80 , debido a que este era un concepto que se consideraba desterrado del ámbito de la enseñanza gracias al auge que tomaron las ciencias de la educación (Zuluaga, 1999). Para lograr un primer acercamiento a lo que se considera práctica pedagógica es necesario usar la noción de práctica planteada por Foucault (1969), desde la arqueología del saber, en donde se entiende este concepto como la compleja relación existente entre instituciones, sujetos y discursos. Adicionalmente, se requiere tener una idea de lo que se entiende por pedagogía. Según Vasco (1990), la pedagogía se puede definir como:

“(...) saber teórico-práctico generado por los pedagogos a través de la reflexión personal y dialogal sobre su propia práctica pedagógica, específicamente en el proceso de convertirla en praxis pedagógica, a partir de su propia experiencia y de los aportes de las otras prácticas y disciplinas que se intersectan con su quehacer". (Vasco, 1990, p.18) 
Por su parte, Zuluaga et al. (2011), de una forma más sencilla y precisa, conciben la pedagogía como una disciplina o saber que conceptualiza, aplica, experimenta y presenta problemas planteados por sí misma o derivados de otras disciplinas.

Complementando un poco la visión de práctica pedagógica presentada anteriormente, Zuluaga (1999), considera que la práctica pedagógica se puede entender como un escenario donde el maestro dispone de todos sus elementos personales y académicos. En lo personal, el maestro tiene en cuenta componentes como el discurso y las relaciones con otros sujetos; en cuanto a lo académico, el maestro debe tener en cuenta el saber disciplinar, el saber didáctico y el saber pedagógico, con el fin de reflexionar acerca de sus fortalezas y debilidades en su quehacer diario para convertir su práctica en un instrumento capaz de generar transformaciones sociales.

Desde una mirada crítica, en donde se reconoce el papel activo del maestro como sujeto promotor de transformación social, investigadores como Saker y Correa (2015), consideran que la práctica pedagógica se debe entender como una:

conjugación de teorías, competencias, didácticas, procedimientos, y estrategias significativas ligadas al proceso educativo que, mediadas por la interacción docente-estudiante, ameritan la permanente indagación, interacción, recontextualización de experiencias y saberes necesarios desde la práctica pedagógica para el rescate de la condición humana. (Saker \& Correa, 2015, p.36)

La importancia de esta nueva mirada que se le ha dado al concepto de práctica pedagógica radica en el reconocimiento del rol que juega el maestro y el impacto que puede tener su labor en la transformación de su realidad, evitando centrarse en la infructífera labor de reproducir contenidos que poco contribuyen al mejoramiento de la calidad educativa. Esta concepción es a la que apunta Gramsci (1973), al hablar acerca de la nueva mirada que se le ha dado a la práctica pedagógica, cuando establece que la relación entre el maestro y el estudiante es una relación activa, de interacciones reciprocas por las cuales todo maestro es al mismo tiempo estudiante y todo estudiante, maestro.

Teniendo en cuenta lo anterior, reducir el concepto de práctica pedagógica solo al quehacer del maestro en el salón de clases corresponde en realidad al concepto de práctica docente y no al de práctica pedagógica. Utilizar ambos términos indistintamente es un error, ya que lo que sucede en el aula o en la escuela en general no es solo el resultado de lo que el maestro quiere, sino la combinación de una serie de variables relacionadas con los sujetos, las instituciones y los discursos. De esta visión de práctica pedagógica se puede inferir que esta constituye una acción social específica, sobre la cual debe reflexionar individual y colectivamente el maestro, que conlleve a convertir a esta en una práctica pedagógica verdadera.

En el presente siglo, las instituciones educativas y los diferentes actores que intervienen o hacen parte de ellas están llamados a reorientar y reorganizar los procesos de formación. Los desafíos, necesidades e intereses que plantea el cambio de época que experimentan los sujetos requieren de maestros responsables, reflexivos y eficientes 
quienes, por medio de la metacognición y el trabajo cooperativo redireccionen y transformen el quehacer educativo. Para Morin (2000), las instituciones educativas deben ser generadoras de cambio y transformación, considerando aspectos esenciales tales como el respeto de las ideas, el sentido humano y lo glocal; por ello, el maestro debe ser un sujeto innovador que genere y propicie espacios que favorezcan la formación continua que tenga en su núcleo la reflexión.

Precisamente en la búsqueda de esta reflexión, la Investigación Acción (IA), se presenta como una de las vías que le permite al maestro investigador reflexionar acerca de su propia práctica pedagógica, a partir de la generación de espacios colaborativos fundamentados en la reflexión y dialogicidad que permitan analizar lo que se hace y cómo se hace en el ámbito educativo, buscando mejorar la práctica. Este ejercicio de valoración acerca de la práctica pedagógica potencia la capacidad reflexiva, investigativa y transformadora del maestro por medio de una formación permanente. Con esto se le da sentido y se ratifica la idea planteada hace algunos años por Carr \& Kemmis (1988), y Stenhouse (1993), quienes resaltan la importancia que tiene la investigación en la práctica pedagógica y el diseño curricular, y la necesidad de que los maestros se conviertan en esos primeros investigadores, quienes trabajando en metanoia se conviertan en generadores de sus propios referentes teóricos desde su práctica pedagógica.

\section{La investigación educativa en el hacer docente}

Algunos de los rasgos característicos de lo que hoy en día se conoce como investigación educativa (conocimiento, intervención, mejoramiento, colaboración), aparecieron con la definición de Investigación Acción (IA), presentada por Lewin (1946). Este autor plantea que la creación de conocimiento científico en el ámbito social es posible si se vincula el trabajo colaborativo de la comunidad que se investiga; en otras palabras, este tipo de investigación promueve la vinculación y participación de los miembros de una comunidad que tienen como objetivo mejorar una situación de la manera más justa y satisfactoria. De manera similar, Carr y Kemmis (1988), se refieren a este tipo de investigación como una forma de autoreflexionar, por parte del maestro, directivo o estudiante, acerca de las acciones educativas y sociales con el fin de mejorar la racionalidad y la justicia de dichas acciones a partir de su comprensión. Estos planteamientos sobre investigación educativa fueron reafirmados por Elliot (1998), quien se refiere a este tipo de investigación como el estudio de una situación social con el fin de mejorar la calidad de la acción dentro de la misma; como una reflexión permanente sobre las acciones humanas y las situaciones sociales que vive el maestro y que busca diagnosticar e intervenir en dichas situaciones de forma efectiva.

Partiendo de lo anterior, es posible definir la investigación educativa como una indagación sistemática, autocrítica y autorreflexiva, fundamentada en el deseo constante por comprender una situación que se considera problemática. Es a la vez una labor y una herramienta constante 
utilizada por el maestro para incidir en las mentes de aquellos actores involucrados. Este tipo de prácticas investigativas busca mejorar la labor del maestro, ya que se pretende desarrollar un trabajo cooperativo, en equipo, con el fin de transformar el contexto o realidad social de los participantes (González et al., 2007).

\section{La metacognición y su relación con la práctica pedagógica}

Durante los últimos años se ha despertado el interés por estudiar el problema del aprendizaje y el desarrollo del conocimiento desde una perspectiva donde el sujeto sea el protagonista principal de su propio proceso de aprendizaje por medio de la reflexión y la autodirección. En otras palabras, se busca que, mediante los procesos formativos, el sujeto aprenda a aprender y a pensar para que construya un aprendizaje significativo y duradero que pueda transferir a los desafíos y retos que emergen en su vida cotidiana. En este sentido, una de las alternativas que permite potenciar la autodirección, el autocontrol y la autoconsciencia sobre el proceso de aprendizaje del sujeto es la metacognición.

Ahora bien, equé se entiende por metacognición? De acuerdo con Glaser (1994), Flavell (1976) y Carretero (2001), este concepto puede ser abordado desde dos aristas distintas: en primera instancia, se relaciona con el conocimiento que el sujeto tiene y construye acerca de la manera como funciona cognitivamente el mismo. Por otra parte, se refiere al proceso de supervisión y regulación de los diferentes procesos que intervienen en la actividad cognitiva cuando el sujeto desarrolla una actividad, una tarea o busca lograr una meta. Es así como se pude establecer una distinción entre el carácter declarativo o conocimiento metacognitivo (saber qué), y el carácter procedimental o control metacognitivo (saber cómo), que definen la naturaleza y la esencia de la metacognición.

En lo que respecta al carácter declarativo de la metacognición, se pueden distinguir tres tipos de conocimiento: la persona, la tarea y las estrategias. En el caso de la persona, se refiere al conocimiento que el sujeto tiene de sí mismo como aprendiz, al conocimiento de sus fortalezas, debilidades y demás características personales. En cuanto a la tarea, hace referencia al conocimiento de los propósitos y las características que hacen que la misma sea sencilla o un poco más compleja de resolver. Y, finalmente, el conocimiento de estrategias concernientes a las potenciales alternativas que le permiten al sujeto desarrollar una tarea de la manera más sencilla y efectiva.

Considerando lo mencionado hasta aquí, lograr que el sujeto aprenda a aprender y además sea capaz de auto-dirigir su proceso de aprendizaje, es una necesidad que no se puede desconocer; por lo tanto, la escuela debe hacer el aporte necesario para que el sujeto se convierta en un aprendiz autónomo en el transcurrir de toda su vida.

Hasta este punto se ha hablado de la metacognición y de la importancia que tiene la misma en los procesos formativos como una forma de responder a las exigencias y necesidades que propone el cambio de época que experimenta el ciudadano del presente siglo. Sin embargo, 
es perentorio precisar cuál es la ruta a seguir para el desarrollo de la metacognición en el sujeto y el rol que tiene el maestro en este proceso. Para contribuir al desarrollo de la metacognición en el estudiante, el primer paso es tener maestros metacognitivos. Si el maestro no redirecciona su práctica pedagógica a partir de sus propias reflexiones, de sus fortalezas y oportunidades de mejora, atendiendo su conocimiento y control metacognitivo, difícilmente podrá orientar un proceso que enseñe a aprender al estudiante y, por ende, no podrá ayudar a potenciar el carácter declarativo y procedimental que caracteriza a la metacognición.

\section{Práctica pedagógica metacognitiva}

Teniendo en cuenta todo lo que se ha planteado hasta aquí, resulta conveniente recordar el carácter complejo del proceso enseñanzaaprendizaje, si se analiza desde el punto de vista del maestro, el estudiante, la familia, la institución educativa, entre otros aspectos. Se requiere, entonces, que el maestro comience a ocupar un rol importante en el desarrollo del conocimiento y el control metacognitivo. Esta motivación es la que le da vida a lo que aquí se le denomina práctica pedagógica metacognitiva, la cual hace referencia a una categoría emergente que visiona a la práctica como un accionar que contribuye al desarrollo de la metacognición en el estudiante. Este tipo de prácticas pedagógicas se puede potencializar a partir de lo que Lin (2001), denomina metacognición adaptativa, que no es más que la adaptación propia del individuo y del ambiente como una forma de hacer más adecuada la instrucción metacognitiva por parte de quien enseña y responder de esta manera a la amplia gama de particularidades que se pueden encontrar en un ambiente de formación. Es así como una práctica pedagógica metacognitiva adaptativa podría facilitar el proceso de religación entre la diversidad de factores que intervienen en el proceso de enseñanzaaprendizaje, la reflexión, el trabajo cooperativo, la dialogicidad y la investigación del maestro dentro de su propia práctica pedagógica (ver esquema $\mathrm{N}^{\circ} 1$ ).

Es evidente que la educación requiere ser resignificada en su estructura, evitando la linealidad y priorizando la dialéctica, las relaciones y las conexiones propias del mundo complejo que habitamos. Una forma de lograr el objetivo planteado anteriormente es por medio del desarrollo de prácticas pedagógicas metacognitivas, ya que estas facilitan y generan las condiciones necesarias para que el maestro investigue sobre su propia práctica, con el fin de potencializar los procesos de transformación social. 


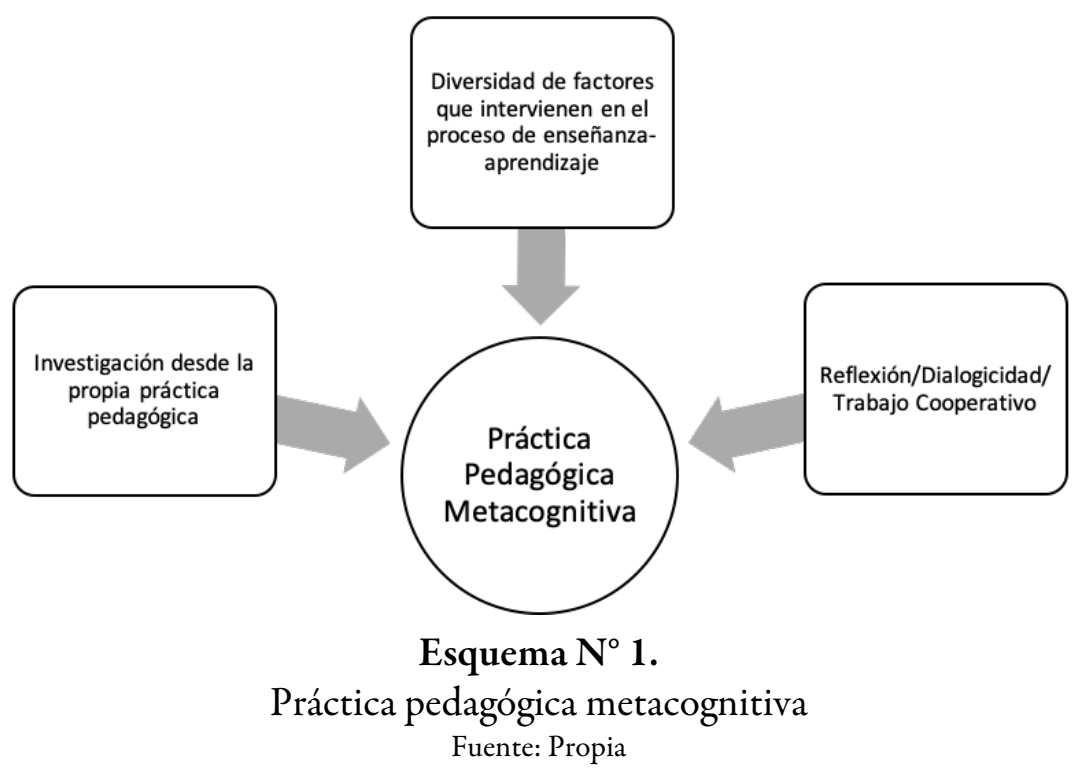

\section{Características principales de la práctica pedagógica metacognitiva}

Tiene su génesis en el carácter complejo del mundo:

Vivimos en un mundo caracterizado y permeado por la complejidad; González (2018), plantea que la realidad en la que se habita es compleja, religada y transdisciplinar; no se puede entender el mundo en el que habitamos desde una mirada diferente a la compleja.

Por lo tanto, nos encontramos ante la necesidad de desarrollar un pensamiento que sea igualmente complejo, tal como lo plantea Edgar Morin. Hoy en día, continuar con un pensamiento que tienda a simplificarlo todo resulta insuficiente para comprender las incertidumbres que emergen a diario. La realidad de nuestro mundo requiere de prácticas pedagógicas que tengan presente esta realidad, su multidimensionalidad y todas las incertidumbres que van emergiendo.

Es dinámica:

La práctica pedagógica metacognitiva se caracteriza por su naturaleza no lineal y anti-estática. Se refiere a un tipo de práctica que se transforma en la medida en que surgen nuevas necesidades y exigencias; es una práctica que, ante todo, contribuye a recontextualizar y transformar realidades a partir de ese carácter dinámico.

Requiere de investigación constante:

La investigación es un elemento base de la práctica pedagógica metacognitiva. Es en el ámbito escolar y mediante la interacción entre el maestro y los estudiantes donde se pueden generar incertidumbres y, al mismo tiempo, alternativas o posibilidades de hacer ajustes y mejoras relacionados con la forma como se enseña y como aprenden los estudiantes, conociendo sus intereses, fortalezas y oportunidades de mejora. Estos espacios potencian ese espíritu investigador del maestro que debe ser impulsado al máximo. En este laboratorio natural de múltiples interacciones sociales, el maestro investigador tendrá tanto aciertos como 
desaciertos que son necesarios para transformar y mejorar la práctica pedagógica.

Es reflexiva:

La reflexión en la práctica pedagógica metacognitiva es un elemento de suma importancia, ya que este elemento le permite al maestro repensarse de forma continua sobre cada una de sus acciones, convirtiéndose en una especie de autoevaluación de su propia práctica con el propósito de resignificarla.

Es dialógica:

La dialogicidad es un elemento complementario de la reflexión que permite analizar de forma colectiva la práctica pedagógica desde una mirada crítica y colaborativa en pro de un objetivo principal que es dinamizar, transformar y mejorar el accionar del maestro.

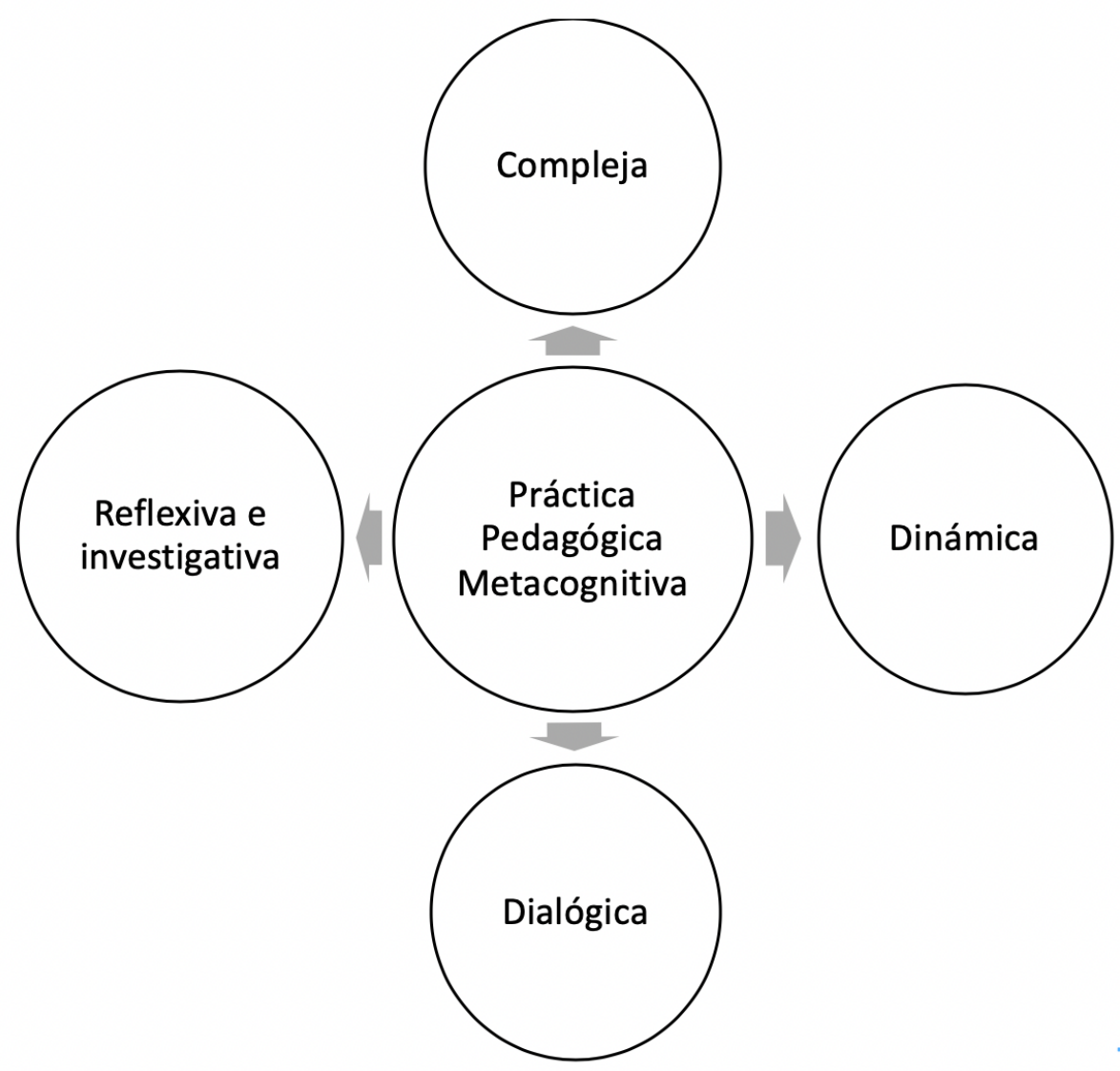

Esquema 2.

Características principales de la práctica pedagógica metacognitiva Fuente: elaboración propia, 2019.

\section{El maestro y el estudiante en la práctica pedagógica metacognitiva}

\section{Los maestros}

En lo que respecta al rol del maestro, es importante destacar que este cumple el papel de modelador y, a la vez, experto al momento de guiar 
a los estudiantes en el uso de herramientas cognitivas y metacognitivas. El maestro es quien de manera explícita muestra cómo se llevan a cabo procesos de pensamiento de tipo metacognitivo, a fin y efecto de que los estudiantes reconozcan y desarrollen estas habilidades. De la misma manera, es el maestro quien por medio de procesos de reflexión y diálogo con otros pares investigará y tomará decisiones acerca de qué habilidades enseñará y cuál será la mejor manera de hacerlo; lo hará teniendo en cuenta las características de sus estudiantes, su contexto, sus intereses y sus necesidades particulares.

Por otro lado, en una práctica pedagógica metacognitiva los maestros están llamados a describir de manera explícita y clara, preferiblemente mediante ejemplos cotidianos, la manera como los estudiantes pueden usar su cerebro ante determinadas situaciones a partir del autoconocimiento y la autoconsciencia que logran tener acerca de sus estilos de aprendizaje.

En relación con la lectura, siempre que exista la posibilidad, el maestro les brindará a los estudiantes la oportunidad de que elijan lo que les gustaría leer y los temas acerca de los que quisieran aprender o profundizar. El resultado más significativo producto de este tipo de prácticas es despertar el interés y mantener la motivación hacia el aprendizaje de diversos temas de estudio en una proyección a largo plazo, lo que para el caso de la lectura se vería reflejado en la creación de una cultura lectora por placer.

Además de la instrucción explícita, la práctica pedagógica metacognitiva comprende tres etapas adicionales complementarias que son las que definirán la formación de estudiantes metacognitivos; estas etapas son la práctica guiada, la práctica cooperativa, para finalmente desarrollar una práctica individual.

Finalmente, toda práctica pedagógica que tenga en su esencia la metacognición se preocupa por tener presente en todo tipo de actividad elementos básicos de un proceso de aprendizaje significativo y orientado al desarrollo de la autodirección en el estudiante; estos elementos incluyen la planificación, la supervisión, el control y la evaluación de las diferentes acciones que se emprenden.

\section{El estudiante}

El éxito académico de los estudiantes está directamente relacionado con su capacidad de pensar, analizar críticamente, tomar decisiones y reflexionar sobre su propio proceso de aprendizaje. Sin embargo, estas son características que se desarrollan en un estudiante cuando vivencia prácticas pedagógicas con estas mismas características. En otras palabras, no podemos pretender formar estudiantes metacognitivos si no se tiene al frente un maestro metacognitivo que oriente este tipo de procesos.

Cuando esto sucede, se contribuye a la formación de estudiantes responsables, organizados, comprometidos, críticos, reflexivos, que constantemente hacen seguimiento a las rutas y estrategias de aprendizaje que más les favorecen. 
Un estudiante que vivencia prácticas pedagógicas metacognitivas es un sujeto capaz de tomar decisiones de manera autónoma y que, incluso, disfruta enseñar o guiar a otros estudiantes.

\section{A modo de síntesis}

La educación como espacio de interacción social requiere de sujetos pensantes, críticos y transformadores capaces de trascender la materialización de las acciones que comprenden una práctica educativa. El cambio de época que estamos experimentando necesita de maestros capaces de promover el desarrollo humano integral. Para ello, uno de los principales retos que tiene el maestro en este mundo permeado por la complejidad es lograr hacer desobediencia epistemológica en cuanto al carácter estático que asumen algunos conceptos. Como educadores, la complejidad de la vida nos invita y nos reta, al mismo tiempo, a movilizar el pensamiento, los saberes, a plantearnos no solo sombras, sino también aquello que es posible y que nos permita comprender mejor nuestras realidades desde nuevas construcciones epistemológicas y teóricas.

En este sentido, es posible afirmar que la práctica pedagógica no es solo un campo de acción; es también sentir, es hacer; es un campo de pensamiento que tiene y ha tenido a lo largo de la historia un carácter dinámico. El concepto, la concepción y el ejercicio de la misma, ha evolucionado y continuará haciéndolo gracias a la actitud de optimismo de los maestros como investigadores y transformadores, tanto de su propia práctica como de sus realidades.

La práctica pedagógica concebida como la compleja relación existente entre instituciones, sujetos y discursos requiere ser vista hoy con una mirada crítica que religue elementos como teorías, procedimientos, estrategias, tanto para la movilización de los saberes como para la evaluación, sumado a políticas educativas y al análisis de los niveles de realidad a los que se enfrenta día a día tanto el maestro como el estudiante, elementos todos que de forma permanente sean cuestionados e investigados; en donde la dialogicidad y la interacción entre los sujetos que intervienen en ella (maestros, estudiantes, familia, sociedad), sean la fuente principal de indagación, reflexión, recontextualización y transformación de esas realidades.

La importancia de la nueva mirada a dicho concepto radica en el reconocimiento del rol que juega el maestro y el impacto en la formación y transformación de los sujetos, evitando centrarse en la infructífera labor de reproducir contenidos que poco contribuyen a la formación integral y que, a su vez, reduce el concepto de práctica pedagógica solo al quehacer del maestro en el salón de clases, lo que corresponde en realidad al concepto de práctica docente. Utilizar ambos términos indistintamente es un error, ya que lo que sucede en el aula o en la escuela en general no es solo el resultado de lo que el maestro quiere, sino la combinación de una serie de variables relacionadas con los elementos descritos en líneas anteriores. De esta visión de práctica pedagógica se puede inferir que esta constituye una acción social específica sobre la 
cual debe reflexionar individual y colectivamente el maestro, que conlleve a convertir a esta en una práctica pedagógica verdadera. En palabras de Morin (2000), las instituciones educativas deben ser generadoras de cambio y transformación, considerando aspectos esenciales tales como el respeto de las ideas, el sentido humano y lo glocal.

\section{REFERENCIAS}

1. Berstein, B. (1998). Pedagogía, control simbólico e identidad. Teoría, investigación y critica. Madrid: Morata.

2. Bourdieu, P. (1987). Los tres estados del capital cultural (Trad. M. Landesmmann). Sociológica, 2(5), 11-17.

3. Carr, W., \& Kemmis, S. (1988). Teoría Crítica de la Enseñanza. La investigación-acción en la formación del profesorado. Ediciones Martínez Roca.

4. Carretero, M. (2001). Metacognición y educación. Aique.

5. Cerdá, M. (2001). Nosotros los maestros. Concepciones de los docentes sobre su quehacer. México: Universidad Pedagógica Nacional.

6. Elliot, J. (1998). La investigación Acción en Educación. Madrid: Morata

7. Flavell, J. (1976). Metacognitive aspects of problem solving. En: Resnik, L. (Ed.). The nature of intelligence (231-235). Hillsdale, N.J.: Erlbaum.

8. Foucault, M. (1969). La arqueología del saber. Editorial Siglo XXI

9. Freire, P. (1982). La educación como práctica de la libertad. Brasil: Ediciones Siglo XXI.

10. Giddens, A. (1986). La constitución de la sociedad: Bases para la teoría de la estructuración. Buenos Aires: Amorrortu Editores.

11. Glaser, R. (1994). Learning theory and instruction. En: D'Ydewalle, G., Eelen, P. y Bertelson, B. (Eds.). International perspectives on psychological science. Hillsdale, N.J.: Erlbaum

12. González, J. (2018). Pensamiento Religado: Pensar para religar. Prisa Ediciones.

13. González, N., Zerpa, M., Gutiérrez, D., \& Pirela, C. (2007). La investigación educativa en el hacer docente. Laurus, 13(23), 279-309.

14. Gramsci, A. (1973). La alternativa pedagógica. Nova Terra.

15. Lewin, K. (1946). Action Research and Minority Problems. Journal of Social Issues, 2, 34-46. https://doi.org/10.1111/j.1540-4560.1946.tb02295.x

16. Lin, X. (2001). Reflective adaptation of a technology artifact: A case study of classroom change. Cognition \& Instruction, 19, 395-440.

17. Morín, E. (2000). Los siete saberes necesarios para la educación del futuro. Ministerio de Educación Nacional. Colombia.

18. Rockwell, E., \& Ezpeleta, J. (1986). La escuela, relato de un proceso de construcción inconcluso. Documento DIE 1. México: IPN

19. Rockwell, E., \& Mercado, R. (1989). La escuela, lugar del trabajo docente. Descripciones y debates. México: Cuadernos de Educación. DIE / CINVESTAV / IPN

20. Saker, J., \& Correa, C. (2015). Saber y Práctica Pedagógica: Aulas abiertas a la investigación educativa. Ediciones Universidad Simón Bolívar. 
21. Stenhouse, L. (1993). La investigación como base de la enseñanza. Madrid: Morata.

22. Tamayo, J. (2017). La práctica pedagógica como categoría de análisis: Acercamientos desde su construcción como objeto de investigación. Congreso Nacional de Investigación Educativa. San Luis, Potosí, México.

23. Vasco, C. (1990). Reflexiones sobre pedagogía y didáctica. Serie pedagogía y currículo. Ministerio de Educación Nacional. Colombia.

24. Zuluaga, O. (1999). Pedagogía e Historia. La historicidad de la pedagogia, la enseñanza, un objeto de saber. Siglo del Hombre Editores, Anthropos.

25. Zuluaga, O., Echeverri, A., Martínez, A., Restrepo, S., \& Quiceno, H., (2011) Educación y Pedagogía: Una diferencia Necesaria. En: O. Zuluaga, A. Echeverri, A. Martínez, H., Quiceno, J., Sáenz, \& Álvarez, A. (Eds.). Pedagogia y Epistemología (21-40). Bogotá: Editorial Magisterio. 